\title{
Strain Relief of Heteroepitaxial bcc-Fe(001) Films
}

\author{
G. Wedler and C. M. Schneider \\ Research Centre Jülich, Institute of Solid State Research, Electronic Properties, D-52425 Jülich, Germany
}

A. Trampert and R. Koch

Paul-Drude-Institut für Festkörperelektronik, Hausvogteiplatz, 5-7, D-10117 Berlin, Germany

(Received 8 June 2004; published 29 November 2004)

\begin{abstract}
The strain relief of heteroepitaxial bcc-Fe(001) films, deposited at 520-570 K onto $\mathrm{MgO}(001)$, has been investigated by scanning tunneling microscopy. In accordance with real-time stress measurements, the tensile misfit strain is relieved during coalescence of flat, mainly 2-3 monolayers (ML) high Fe islands at the high thickness of $\sim 20 \mathrm{ML}$. To accommodate the misfit between merging strain-relaxed islands, a network of $\frac{1}{2}[111]$ screw dislocations is formed. A strong barrier for dislocation glide - which is typical for bcc metals - is most likely responsible for the big delay in strain relief of $\mathrm{Fe} / \mathrm{MgO}(001)$, since only the elastic energy of the uppermost layer(s) is available for the formation of an energy-costly intermediate layer.
\end{abstract}

DOI: 10.1103/PhysRevLett.93.236101

The relaxation of strain is an important and often crucial issue in heteroepitaxy, where strain values of a few percent are quite common due to lattice mismatch between film and substrate. Strain of that order corresponds to huge stress, which typically exceeds the tensile strength of the bulk phase by at least one order of magnitude. Therefore mismatch strain jeopardizes the structural stability of the films and thus the reliable operation of devices. During film growth, mismatch strain typically is relaxed after deposition of one or a few pseudomorphic atomic layers, by one of the two mechanisms: (a) Formation of dislocation-free 3D islands on top of a continuous wetting layer [1-3] via Stranski-Krastanow growth [4]. This mechanism received considerable attention in semiconductor heteroepitaxy, because it offers a means to fabricate selforganized quantum dot arrays for optoelectronics; examples are $\mathrm{Ge} / \mathrm{Si}(001)$ [1], $\operatorname{InAs} / \mathrm{GaAs}(001)$ [5], or $\mathrm{PbSe} / \mathrm{PbEuTe}(001)$ [6]. (b) Alternatively, misfit dislocations may be inserted directly into the pseudomorphic film [7-9], i.e., without nucleating 3D islands, as observed for InAs/GaAs(001) [10], $\mathrm{PbTe} / \mathrm{PbSe}(001)$ [11], or $\mathrm{Ni} / \mathrm{Cu}(001)$ [12]. When atomic rows are inserted or omitted already in the first atomic layer (i.e., directly at the interface) - as found for $\mathrm{Cu} / \mathrm{Ru}(0001)$ [13,14], $\mathrm{Ni} / \mathrm{Re}(0001)$ [15], or $\mathrm{Ag} / \mathrm{Pt}(111)$ [16] - it is more appropriate to speak of commensurate growth as the films themselves do not contain dislocations. It should be emphasized, however, that also the strain of larger StrankiKrastanow [17,18] or Volmer-Weber [19] islands ultimately is relieved by misfit dislocations.

So far the vast majority of investigations on strain relief deal with epitaxial films belonging to the fcc crystal class, whereas the strain relaxation of bcc films is less well understood. A major difference between fcc and bcc arises from the different symmetry of the shortest translation vectors and hence the shortest Burgers vectors for
PACS numbers: 68.55.Ac, 68.35.Gy, 68.37.Ef, 81.15.-z

perfect dislocations: it is twofold for the fcc $(a / 2)\langle 110\rangle$ vector and threefold for the bcc $(a / 2)\langle 111\rangle$ vector. Since the dislocation cores in bcc are farther spread out, the Peierls energy is increased, and accordingly also the activation barrier for dislocation glide. This explains, e.g., the low mobility of $(a / 2)\langle 111\rangle$ screw dislocations in plastic deformation of bec bulk materials [20] and may govern also nucleation and glide of dislocations in bcc films.

Here we report on the strain relief of bcc-Fe(001) films deposited onto $\mathrm{MgO}(001)$ - a heteroepitaxial system that is extraordinary in several respects: (i) $\mathrm{Fe} / \mathrm{MgO}(001)$ is one of the few metal on insulator systems that grows layer by layer at certain conditions as predicted by theory [21] and verified experimentally [22-24]. In that context it has become a model system for the investigation of magnetic [21,24,25] and transport properties [26] of ultrathin metal films. (ii) Thickness dependent stress measurements revealed that the tensile misfit strain $(3.5 \%)$ is not relieved before a thickness of 2-3 nm (15-20 atom layers) is reached (cf. Fig. 1). This thickness indeed is rather high and exceeds significantly the critical layer thickness at which strain relief by misfit dislocations becomes energetically favorable $(0.85$ and $1.25 \mathrm{~nm}$ ac-

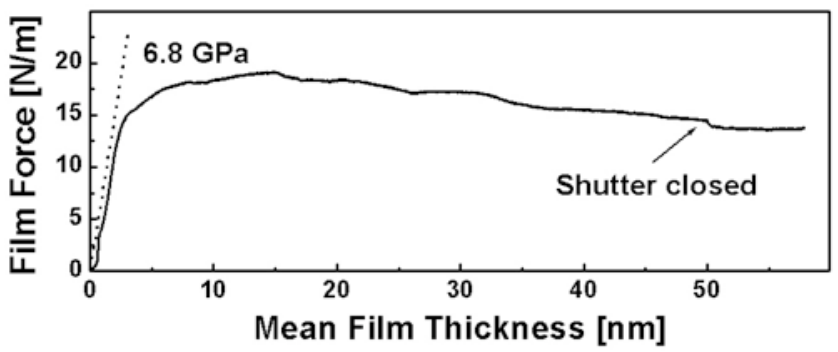

FIG. 1. Film forces (i.e., integral forces in films of unit width) as a function of the mean film thickness measured in real time during the deposition of $\mathrm{Fe}$ onto $\mathrm{MgO}(001)$ at $520 \mathrm{~K}$. 
cording to the formulas of Refs. [7,8], respectively). (iii) Since Fe grows in its bcc modification on a substrate that is fcc, the transmission of dislocations from the film to the substrate and/or vice versa is suppressed. Therefore $\mathrm{Fe} / \mathrm{MgO}(001)$ is an ideal system to study the mechanisms of strain relief in a bcc film itself. Our in situ STM (scanning tunneling microscopy) results identify the merging of 2-3 monolayer thin islands on top of a $2-3 \mathrm{~nm}$ thick continuous $\mathrm{Fe}$ layer as the predominant mechanism for strain relaxation, in the course of which a network of screw dislocations is formed.

The experiments were performed in a UHV chamber (base pressure $<10^{-10}$ mbar) equipped with an Omicron UHV-STM. Prior to mounting into the UHV chamber the $\mathrm{MgO}(001)$ substrates [27] were baked for about $10 \mathrm{~h}$ at $1300 \mathrm{~K}$ in a stream of oxygen at atmospheric pressure; to prevent desorption of oxygen they were only shortly degassed in UHV at 520-570 K before the film deposition. Fe was evaporated from a rod heated by a tungsten filament at a deposition rate of $0.008 \pm 0.001 \mathrm{~nm} / \mathrm{s}$. The pressure during deposition was better than $1 \times 10^{-9}$ mbar. After preparation the samples were cooled to room temperature and transferred to the STM.

To facilitate the further discussion, the stress behavior of $\mathrm{Fe} / \mathrm{MgO}(001)$ prepared at $520 \mathrm{~K}$ is illustrated in Fig. 1. As shown previously [23], the film stress $(\sigma)$ at the beginning of deposition is dominated by the misfit of $3.5 \%$. Accordingly, the film forces $(F / w=\sigma t$ with $w$ and $t$ denoting film width and thickness, respectively) increase nearly linearly with the film thickness. The initial slope corresponds to tensile stress of about $6.8 \mathrm{GPa}$ in good agreement with the maximum misfit stress at $520 \mathrm{~K}(6.8 \mathrm{GPa}$, indicated by dashed line). At a thickness of $2-3 \mathrm{~nm}$ the slope of the force curve decreases, indicating relaxation processes that suppress the further transmittance of misfit strain. Notice, however, that the tensile force component built up initially is conserved up to high thicknesses $(>100 \mathrm{~nm}$; see Ref. [23]), thus pointing to a strongly stressed Fe layer at the interface.

Our STM study indeed reveals continuous Fe films above thicknesses of $1.3 \mathrm{~nm}$; at lower thickness steps of the insulating substrate inhibit long-range conductivity necessary for STM investigations. Figure 2(a) displays an STM top view image of a $7.5 \mathrm{~nm}$ thick Fe film deposited at $570 \mathrm{~K}$ onto $\mathrm{MgO}(001)$. According to Fig. 1, at this thickness the Fe film is in a stage where the relief of misfit strain is in progress. Interestingly, the entire film surface is patterned by a dense array of square islands with heights of about $1 \mathrm{~nm}$ [cf. line scan of Fig. 2(a)], which sit on top of a continuous Fe layer of about $6 \mathrm{~nm}$. The average island length is about $28 \mathrm{~nm}$. Each island contains at least one, frequently two (right- and lefthanded), screw dislocations [cf. inset of Fig. 2(a)]. Our STM investigations therefore impressively disclose that in the case of bcc-Fe(001) films - in contrast to fcc films investigated previously - a large number of screw dis-
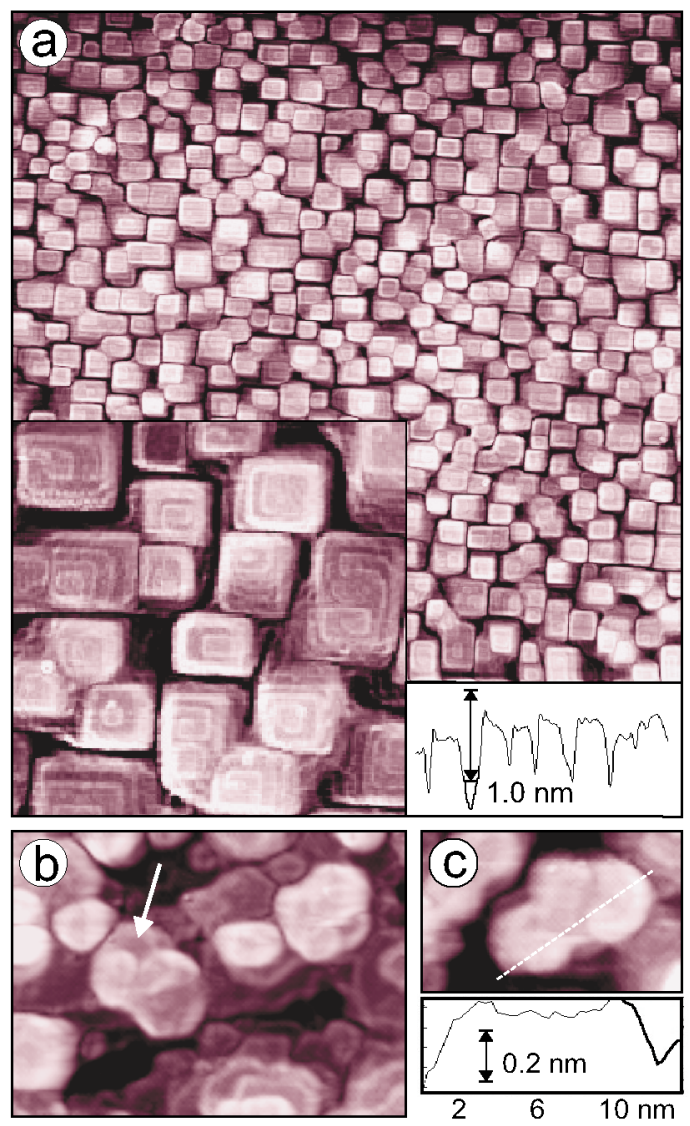

FIG. 2 (color online). Strain relaxation in $\mathrm{Fe} / \mathrm{MgO}(001)$ : (a) $600 \times 600 \mathrm{~nm}^{2} \mathrm{STM}$ image of a $7.5 \mathrm{~nm}$ Fe film deposited at $570 \mathrm{~K}$; the entire film surface is patterned by a dense array of square islands with heights of about $1 \mathrm{~nm}$ (cf. line scan) which sit on top of a $6 \mathrm{~nm}$ thick continuous layer; each island contains at least one, frequently two (right- and left-handed), screw dislocations. Inset: $180 \times 180 \mathrm{~nm}^{2}$ zoom displaying the screw dislocations in more detail; bias voltage $U_{T}=80 \mathrm{mV}$; tunneling current $I_{T}=1 \mathrm{nA}$. (b) $36 \times 26 \mathrm{~nm}^{2} \mathrm{STM}$ image of a $5 \mathrm{~nm}$ Fe film deposited at $520 \mathrm{~K}$ illustrating the formation of screw dislocations (arrow) during island coalescence; for details see text; $U_{T}=250 \mathrm{mV} ; I_{T}=0.35 \mathrm{nA}$. (c) Respective $19 \times 12 \mathrm{~nm}^{2}$ STM image showing an island with borders raised by $\sim 0.03 \mathrm{~nm}$ because of lattice contraction (cf. Fig. 3); single scan is along dashed line in the top view; $U_{T}=250 \mathrm{mV} ; I_{T}=$ $0.35 \mathrm{nA}$.

locations is formed upon strain relaxation. At thicknesses below $2.5 \mathrm{~nm}$ no screw dislocations were detected.

In Fig. 3 we sketch a realistic mechanism for the insertion of screw dislocations into a growing (001) film. The bottom layer in Fig. 3, illustrated by dark spheres, represents the uppermost (001) plane of the biaxially expanded continuous Fe layer ( $\sim 6 \mathrm{~nm}$ thick). For reasons of presentation we increased the misfit of the sphere model to $\sim 11 \%$, i.e., requiring an additional row every nine rows for complete relief of the misfit strain. For comparison, in $\mathrm{Fe} / \mathrm{MgO}(001)$ with a misfit of $3.5 \%$ insertion of an additional row every 29 lattice distances $(\sim 9 \mathrm{~nm})$ is sufficient. The two 2D islands in Fig. 3growing on top of the strained (001) plane - have relaxed 


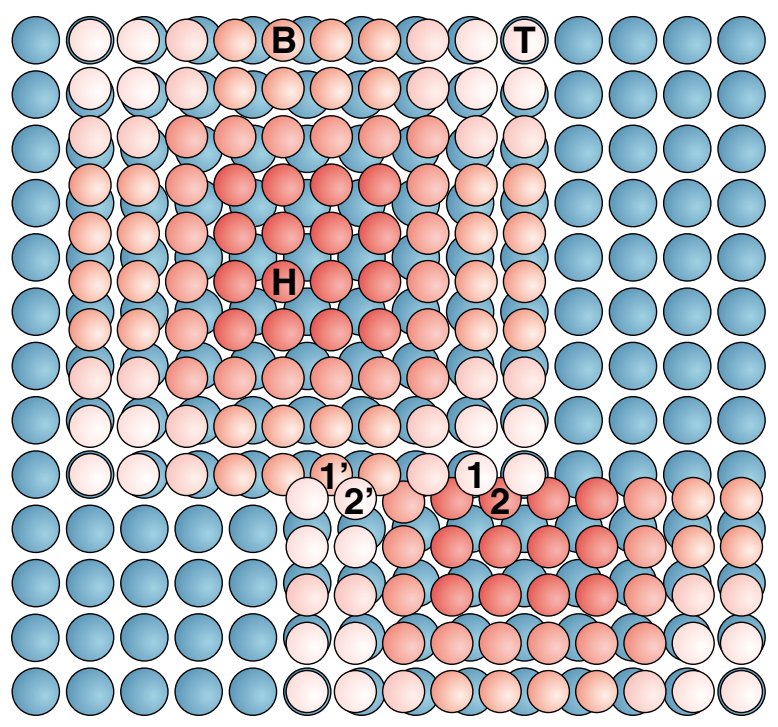

FIG. 3 (color online). Sphere model (top view) illustrating the mechanism for strain relaxation in $\mathrm{Fe} / \mathrm{MgO}(001)$ : The bottom layer (dark spheres) represents the uppermost (001) plane of the expanded and thus highly strained continuous $\mathrm{Fe}(001)$ layer. For reasons of presentation the misfit is increased to $\sim 11 \%$ in the sphere model, i.e., requiring an additional row every nine rows for complete strain relief. Upon coalescence of the two strain-relaxed 2D islands (bright spheres) a screw dislocation is formed in the contact region (for details see text).

the strain. Because of the reduced lattice spacing, only the atoms in the center of the square upper island of Fig. 3 sit in the fourfold hollow sites of the bcc lattice (indicated by $\mathrm{H})$. Atoms near the step edges are shifted to energetically less favorable bridge sites (B) as well as to on top sites in the four corners (T). In the lower part of Fig. 3 there is a second $2 \mathrm{D}$ island which is coalescing with the upper island. At the contact region atoms of the lower island (marked by 2) have moved beneath the edge and the corner atoms of the upper island (marked by 1), thus pushing them upwards onto top sites of the bcc lattice. Because of the strain relaxation in the lower island, a reversed situation is observed farther to the left in Fig. 3, where atoms of the square island lie underneath (i.e., $1^{\prime}$ below $2^{\prime}$ ). A closer look reveals that the restructuring of the contact region has led to two screw dislocations with $(a / 2)\langle 111\rangle$ Burgers vectors, suggesting that island coalescence may provide a natural way to insert screw dislocations in $\mathrm{Fe} / \mathrm{MgO}(001)$.

That island coalescence indeed plays a crucial role in the formation of screw dislocations is supported by the STM image of Fig. 2(b). It shows a $5 \mathrm{~nm}$ thick Fe film on $\mathrm{MgO}(001)$, deposited at the lower temperature of $520 \mathrm{~K}$. Because of the reduced surface diffusion at $520 \mathrm{~K}$ the island shapes are less regular and therefore still contain information of the island coalescence. The island marked by the arrow-being only one example out of manyhas emerged from coalescence of two smaller islands. In agreement with the model of Fig. 3 two screw dislocations have formed, with their cores lying in the boundary region. Notice also the island of Fig. 2(c) with borders that are raised by $\sim 0.03 \mathrm{~nm}$, thus indicating lattice contraction as illustrated in Fig. 3.

Further insight is obtained from the energetics of strain relief and dislocation formation. The elastic energy density $E_{\mathrm{el}}$ of a $\mathrm{Fe}(001)$ film strained biaxially by misfit $\varepsilon_{\mathrm{MF}}$ is given by

$$
E_{\mathrm{el}}=\frac{\left(C_{11}-C_{12}\right)\left(C_{11}+2 C_{12}\right)}{C_{11}} \varepsilon_{\mathrm{MF}}
$$

with $C_{i j}$ being the elastic constants. For an unrelaxed $10 \times 10 \mathrm{~nm}^{2}$ sized $2 \mathrm{D} \mathrm{Fe}$ island a strain energy of $22.3 \mathrm{eV}$ is calculated $(18.2 \mathrm{meV} /$ atom). To estimate the energy of an extended screw dislocation line with Burgers vector $\vec{b}$ we use the common expression for the energy per $b$ [28]:

$$
E_{\mathrm{disl}}=E_{\mathrm{c}}\left(r_{\mathrm{c}}\right)+\frac{K b^{3}}{4 \pi} \ln \left(\frac{R}{r_{\mathrm{c}}}\right)+A\left(d_{i}\right) .
$$

The first term in Eq. (2), $E_{c}\left(r_{c}\right)$, is the energy of the dislocation core. It is nowadays calculated by molecular dynamics or ab initio methods, since close to the core, i.e., $r<r_{c}$, the lattice is severely distorted by the dislocation and a continuum description fails. The second term describes the elastic energy of the dislocation outside of the core with $K$ and $R$ being the corresponding shear modulus and an upper cutoff length, respectively. The term $A\left(d_{i}\right)$ accounts for the long-range elastic interactions between different dislocations at distance $d_{i}$. With $b=$ $\sqrt{3} / 2 a_{\mathrm{Fe}} \quad\left(a_{\mathrm{Fe}}=0.287 \mathrm{~nm}\right), K \cong 70 \mathrm{GPa}, \quad R=10 \mathrm{~nm}$, and inserting a typical value for $r_{\mathrm{c}}=2 b$, the elastic energy is calculated to $1.6 \mathrm{eV} / b$. Typical values for the core energy $E_{\mathrm{c}}$ lie between $1-2.5 \mathrm{eV} / b[29,30]$, for $A\left(d_{i}\right)$ values of $-0.5 \mathrm{eV} / b$ are obtained [29]. In total, we estimate the energy of a screw dislocation in $\mathrm{Fe} / \mathrm{MgO}(001)$ per (001) monolayer (ML) to be about 1-2 eV. With two screw dislocations formed per island, the energy cost of dislocations therefore is by far overcompensated by the gain in elastic energy due to the strain relief.

Because of this large gain in energy, the growth and stability of a highly strained continuous $\mathrm{Fe}(001)$ layer at the interface are-at first sight-quite surprising. As revealed by our real-time stress measurements (see Fig. 1), the high tensile misfit strain of $3.5 \%$ is transmitted up to an unusually large thickness of about $20 \mathrm{ML}$, thus pointing to a strong kinetic barrier for the insertion of dislocations. Moreover, the strain of this layer is not relieved upon further growth, thus indicating a strong barrier for dislocation glide. We note that the existence of this highly strained interface layer is further corroborated by our unsuccessful attempts to prepare thin samples for cross-sectional TEM (transmission electron microscopy). In spite of many years of experience in TEM sample preparation and particularly with $\mathrm{Ag} / \mathrm{MgO}(001)$ [31], we find a large number of microcracks to be injected 
into the substrate upon etch thinning most likely due to the huge residual stress in the Fe film.

According to the model of Fig. 3, strain relief in the 2D islands proceeds by lattice contraction and eventually insertion of two additional atomic rows along [100] and [010], respectively. This means that finally two orthogonal misfit dislocations are formed with respect to the strained $\mathrm{Fe}(001)$ lattice. The misfit between merging islands, on the other hand, is accommodated by screw dislocations. As illustrated in Fig. 3, strain relief leads to serious atomic distortions in a relaxed 2D layer growing atop the strained (001) plane. Hardly any atom is occupying the energetically most favorable fourfold hollow sites of the bcc lattice; the majority of atoms are decisively displaced and even shifted to less favorable bridge and on top sites. The energy of such a distorted intermediate layer, which ultimately separates strained from relaxed Fe, is certainly very large, even in comparison with the elastic energy stored in a strained monolayer. In fact, from our experiments we conclude that the gain in elastic energy from a single layer is smaller than the formation energy of the relaxed intermediate layer. Therefore the initial 2D islands have to reach a certain height (2-3 ML according to Figs. 2(b) and 2(c) in order to accumulate elastic energy. Since upon coalescence the elastic energy of two or more layers is released simultaneously, strain relaxation by the proposed mechanism becomes feasible. We note that a gradual increase of the surface roughness might be the result of a remaining influence of step edge barriers on surface diffusion $[32,33]$.

All in all, our experimental and theoretical results of $\mathrm{Fe} / \mathrm{MgO}(001)$ lead to a conclusive picture of the strain relief in a bcc-metal film. Coalescence of islands of appropriate height is providing an energetically favorable path for the nucleation of dislocations, whereby the misfit between merging islands is accommodated by screw dislocations. Interestingly, there is a pronounced preference for forming screw dislocations which is in clear contrast to the strain relief in fcc films. The strong barrier for dislocation glide - which is typical for bcc metals - is most likely responsible for the big delay in strain relief of $\mathrm{Fe} / \mathrm{MgO}(001)$, since only the elastic energy of the uppermost layer(s) is available for the formation of an energycostly intermediate layer. In fact, strong barriers for dislocation glide might provide a general explanation for the large kinetic barriers to inject dislocations in heteroepitaxial systems.

[1] Y.-W. Mo, D. E. Savage, B. S. Swartzentruber, and M. G. Lagally, Phys. Rev. Lett. 65, 1020 (1990).

[2] J. Tersoff and R. M. Tromp, Phys. Rev. Lett. 70, 2782 (1993).

[3] E. Pehlke, N. Moll, A. Kley, and M. Scheffler, Appl. Phys. A 65, 525 (1997).

[4] E. Bauer, Z. Kristallogr. 110, 372 (1958).
[5] S. Guha, A. Madhukar, and K. C. Rajkumar, Appl. Phys. Lett. 57, 2110 (1990).

[6] G. Springholz, M. Pinczolits, P. Mayer, V. Holy, G. Bauer, H. H. Kang, and L. Salamanca-Riba, Phys. Rev. Lett. 84, 4669 (2000).

[7] J. H. Van der Merwe, J. Appl. Phys. 34, 123 (1962).

[8] J.W. Matthews and A. E. Blakeslee, J. Cryst. Growth 27, 118 (1974).

[9] R. People and J. C. Bean, Appl. Phys. Lett. 47, 322 (1985).

[10] E. Tournié, A. Trampert, and K. H. Ploog, Europhys. Lett. 25, 663 (1994).

[11] G. Springholz and K. Wiesauer, Phys. Rev. Lett. 88, 015507 (2002).

[12] H. E. Inglefield, C. A. Ballentine, G. Bochi, S. S. Bogomolov, R. C. O'Handley, and C.V. Thompson, in Thin Films: Stresses and Mechanical Properties IV, edited by P. H. Townsend, T.P. Weihs, and J. Sanchez, Jr., MRS Symposia Proceedings No. 308 (Materials Research Society, Pittsburgh, PA, 1993), p. 765.

[13] C. Günther, J. Vrijmoeth, R. Q. Hwang, and R. J. Behm, Phys. Rev. Lett. 74, 754 (1995).

[14] J. de la Figuera, K. Pohl, O. Rodriguez de la Fuente, A. K. Schmid, N.C. Bartelt, C. B. Carter, and R. Q. Hwang, Phys. Rev. Lett. 86, 3819 (2001).

[15] M. Stindtmann, M. Farle, T. S. Rahman, L. Benabid, and K. Baberschke, Surf. Sci. 381, 12 (1997).

[16] J. C. Hamilton, R. Stumpf, K. Bromann, M. Giovannini, K. Kern, and H. Brune, Phys. Rev. Lett. 82, 4488 (1999).

[17] F. K. LeGoues, J. Tersoff, M. C. Reuter, M. Hammar, and R. Tromp, Appl. Phys. Lett. 67, 2317 (1995).

[18] X. H. Liu, F. M. Ross, and K.W. Schwarz, Phys. Rev. Lett. 85, 4088 (2000).

[19] J.W. Matthews, in Physics of Thin Films, edited by G. Hass and R.E. Thun (Academic Press, New York, 1967), Vol. 4, p. 137.

[20] A. Seeger and C. Wüthrich, Nuovo Cimento Soc. Ital. Fis. B 33, 38 (1976).

[21] C. Li and A. J. Freeman, Phys. Rev. B 43, 780 (1991).

[22] T. Urano and T. Kanaji, J. Phys. Soc. Jpn. 57, 3403 (1988).

[23] R. Koch, M. Weber, K. Thürmer, and K. H. Rieder, J. Magn. Magn. Mater. 159, L11 (1996).

[24] A. Subagyo, K. Sueoka, K. Mukasa, and K. Hayakawa, Jpn. J. Appl. Phys. 38, 3820 (1999).

[25] G. Wedler, J. Walz, A. Greuer, and R. Koch, Phys. Rev. B 60, R11 313 (1999).

[26] R. Schad, P. Beliën, G. Verbanck, V.V. Moshchalkov, and Y. Bruynseraede, J. Phys. Condens. Matter 10, 6643 (1998).

[27] CrysTec GmbH, crystec@aol.com.

[28] J. P. Hirth and J. Lothe, Theory of Dislocations (John Wiley \& Sons, Inc., New York, 1982).

[29] S. Ismail-Beigi and T. A. Arias, Phys. Rev. Lett. 84, 1499 (2000).

[30] Y. Qi, A. Strachan, T. Cagin, and W. A. Goggard III, Mater. Sci. Eng. A 309-310, 156 (2001).

[31] A. Trampert, F. Ernst, C. P. Flynn, H. F. Fischmeister, and M. Rühle, Acta Metall. Mater. 40, S227 (1992).

[32] K. Thürmer, R. Koch, M. Weber, and K. H. Rieder, Phys. Rev. Lett. 75, 1767 (1995).

[33] J. A. Stroscio, D. T. Pierce, M. D. Stiles, A. Zangwill, and L. M. Sander, Phys. Rev. Lett. 75, 4246 (1995). 